\title{
Novel Use of the Sigmoidoscope to Perform Difficult Papanicolaou Smears
}

\author{
CAPT Robert F. Raspa, MC, USN (Ret.)
}

Obtaining a Papanicolaou smear in obese patients can be a difficult task encountered in Family Practice. In this case, a sigmoidoscope was successfully used to easily obtain a Papanicolaou smear after other methods had failed.

\section{Case Report}

An obese (body mass index of 41) 45-year-old woman presented to clinic to obtain a Papanicolaou smear. Attempts were made to obtain the Papanicolaou smear by the usual method and by an alternative method of placing a glove over the speculum to hold back the vaginal sidewalls. Both methods were unsuccessful and resulted in considerable patient discomfort. The patient, who was a health care professional, readily agreed to try using a sigmoidoscope to provide visualization of the cervix and facilitate obtaining the Papanicolaou smear. After informed consent was obtained, the patient was placed in the lithotomy position and the sigmoidoscope was inserted into the vagina. With minimal air insufflation (less than $150 \mathrm{~mL}$ as 2 to 3 small puffs of air), the cervix was visualized. A wooden Papanicolaou spatula and a cytobrush were individually inserted alongside the sigmoidoscope, and the Papanicolaou smear was obtained while visualization of the cervix was maintained using the scope. Pictures were taken. The process was repeated with a cytobrush to obtain an endocervical sample. The patient experienced no complications and stated the procedure was much more comfortable than using a vaginal speculum. The Papanicolaou smear specimen was adequate for diagnosis and included endocervical cells.

Submitted, revised, 3 February 2003.

From Naval Hospital Jacksonville, Jacksonville, Florida. Address correspondence to CAPT Robert F. Raspa, MC, USN, 2233 Salt Myrtle Lane, Orange Park, FL 32003.

\section{Discussion}

No reports of using a sigmoidoscope to obtain a Papanicolaou smear were found in the literature. Although sigmoidoscopy equipment is expensive, most family physicians have ready access to a unit. The only serious potential complication is air embolus caused by aggressive air insufflation with the scope. Air embolus through vaginal insufflation, although a rare occurrence, has been reported in the literature and usually occurs with pregnant patients. ${ }^{1}$ It is postulated that air can dissect along the fetal membranes and uteroplacental tissue planes and be absorbed by the subplacental venous sinusoids. Therefore, this procedure is contraindicated in pregnancy. Air embolus, a potential occurrence in the nonpregnant patient, is much more rare and it is estimated that well over $250 \mathrm{~mL}$ of air must be forcibly delivered in the vagina before embolism can occur in the nonpregnant patient. ${ }^{2}$

\section{Conclusion}

Using a sigmoidoscope to provide visualization of the cervix to obtain a Papanicolaou smear when more common methods have failed is a simple, effective, comfortable alternative. Family physicians with access to a sigmoidoscope should consider using this instrument in selected nonpregnant patients.

\section{References}

1. Kaiser RT. Air embolism death of a pregnant woman secondary to orogenital sex. Acad Emerg Med 1994; 1:555-8.

2. Fyke FE 3rd, Kazmier FJ, Harms RW. Venous air embolism. Life-threatening complication of orogenital sex during pregnancy. Am J Med 1985;78: $333-6$. 